\title{
Le complexe de Diane dans l'hagiographie chrétienne
} Marie Delcourt

\section{Citer ce document / Cite this document :}

Delcourt Marie. Le complexe de Diane dans l'hagiographie chrétienne. In: Revue de l'histoire des religions, tome 153, ${ }^{\circ} 1$, 1958. pp. 1-33;

doi : 10.3406/rhr.1958.8774

http://www.persee.fr/doc/rhr_0035-1423_1958_num_153_1_8774

Document généré le 03/05/2016 


\section{Le complexe de Diane dans l'hagiographie chrétienne}

Parce que Freud a parlé du complexe d'Edipe, les psychanalystes désignent volontiers de noms mythologiques les tendances qui contrarient l'équilibre de l'âme. L'horreur de certaines femmes pour la condition féminine, c'est Minerve plutôt que Diane qui aurait dû lui donner son parrainage. Diane est vierge et chasseresse, mais elle protège les femmes en couches, tandis qu'Athéna, née sans mère de la tête d'un dieu, se vante dans les Euménides de "pencher toujours du côté des hommes, sauf en ce qui concerne le mariage ". En dehors de cette déesse casquée, les Grecs connaissent plus d'une personnification du complexe de Diane : les Amazones, les Danaïdes, Daphné qui hait les hommes et ne se plaît qu'à la chasse, Kainis qui obtient de Posidon de devenir homme et invulnérable et qui aussitôt divinise sa lance' ${ }^{1}$. Dans la même ligne, Virgile imagine sa Camille, qui servira de modèle aux Clorindes de la Renaissance.

Parmi les romans que contiennent les Vies des saints figurent de curieux récits où se lit, pliée à une éthique nouvelle, une autre forme du complexe de Diane. Des femmes, vierges ou pécheresses repenties, se retirent du monde pour aller vivre, non point comme tant d'autres parmi des religieuses, mais dans un couvent d'hommes, ou parfois en marge du monastère, en se donnant pour des eunuques. Dans d'autres récits, probablement aussi légendaires que les premiers, le

1) Le terme de complexe de Diane vient, je crois, de Charles Baudouin; d'autres parlent de complexe de virilité. Sur les Danaïdes, cf. M. D. Kouretas, La névrose sexuelle des Danaïdes, Rev. franc. de psychanalyse, juill. 1957. - Sur Kainis, cf. $R H R, 1953$, p. 129. - La légende d'Agnodiké, jeune Athénienne qui se déguisa en homme pour pratiquer l'obstétrique (Hygin, fable 274), est colorée par le complexe de Diane, mais le thème de l'ostension sexuelle, qui y figure deux fois, l'apparente à une Baubô. 
travestissement ne dure que le temps de permettre à une fille menacée de fuir un milieu païen et de suivre un maitre élu. Les philologues et les hagiographes qui ont étudié ces histoires, H. Usener, L. Radermacher, le P. H. Delehaye, ont cherché surtout à découvrir les sources d'un thème singulier. Ils ne se sont pas arrêtés à en scruter la signification. Moins encore se seraient-ils avisés de confondre histoire et légende en rapprochant de Thècle et de Pélagie des femmes réelles et plus proches de nous, comme Jeanne d'Arc ou Antoinette Bourignon. Et cependant rien n'enseigne mieux la valeur de certains symboles que de les voir attestés à la fois dans la légende et dans l'histoire, avec des constantes psychologiques que n'altèrent ni les siècles ni le dépaysement.

Un simple inventaire permettra de suivre le thème directeur du travestissement masculin. Pour une légende donnée, un classement chronologique des traditions est généralement impossible. La préhistoire des récits que nous lisons ne nous est pas accessible. Nous résumons ici ce qu'on trouve dans les Acta Sanctorum, en renvoyant, le cas échéant, à la Légende dorée (traduction E. B $<$ runet $>$, 1843).

\section{I. - Saintes ermites ou moines}

1. Pelagia, danseuse à Antioche, est une belle pécheresse que le peuple nomme Margarito à cause de ses joyaux. Convertie par Nonnus, ascète de la règle de saint Pacôme, elle reçoit le baptême et, avec l'assentiment de Nonnus, revêt un froc fait de poils. Elle se rend à Jérusalem où, enfermée dans une étroite cellule sur le mont des Oliviers, elle fait pénitence sous le nom de frater Pelagius monachus et eunuchus. Épuisée par les austérités, elle meurt au bout de trois ans (fête le 8 octobre; Acta Sanct., oct. IV, p. 248; Légende dorée, II, p. 176).

2. Margarita, fille de parents nobles et déjà fiancée par eux, a une telle horreur du mariage qu'elle s'enfuit de la chambre nuptiale en habits masculins et les cheveux coupés pour se réfugier dans un monastère sous le nom de Pelagius. 
Ses qualités lui valent d'être nommée prieur d'un couvent de filles. La sœur portière se trouve enceinte et accuse le prieur qui est chassé. Margarita va vivre une austère vie d'ermite dans une caverne où, avant de mourir, elle révèle à l'abbé à la fois son sexe véritable et son innocence (Acta, juillet IV, p. 287 ; oct. IV, p. 24 ; Légende dorée, II, p. 178). 3. Athanasia et Andronicos d'Antioche, confesseurs. Après la naissance de leurs deux enfants, ces époux chrétiens décident de vivre comme frère et sœur ; plus tard, leurs enfants étant morts le même jour, de tout donner aux pauvres, et d'aller en pèlerinage à Jérusalem avant d'adopter la vie monastique. Après douze ans, Andronicos obtient de son abbé la permission de retourner à Jérusalem. Athanasia de son côté fait de même, en vêtements masculins. Ils se rencontrent en Égypte sans que le mari reconnaisse sa femme. De retour à Alexandrie, elle entre sous le nom d'Athanasios dans le couvent de son mari et y vit douze ans, jusqu'à ce que la mort les enlève en même temps (fête le 9 oct., Acta, oct. IV, p. 997).

4. Marina est la fille d'un Bithynien nommé Eugenios qui, resté veuf, souhaite entrer au couvent. Marina obtient de lui qu'il lui coupe les cheveux et l'emmène au monastère où, sous le nom de frère Marinos, elle reste après la mort de son père. A cause de sa voix grêle et de son menton nu, les moines la considèrent comme un eunuque. La fille d'une auberge voisine se trouve enceinte et accuse Marinos de l'avoir séduite. Expulsée du couvent, elle mène aux environs une vie de pénitence tout en soignant l'enfant que le père de la fille lui a mis dans les bras. L'abbé ému de pitié finit par accueillir de nouveau Marinos qui fait les plus humbles besognes jusqu'au jour où on la trouve morte dans sa cellule ; la fille confesse son mensonge (fête le 17 juillet, Acta, juill. IV, p. 278 ; Légende dorée, I, p. 149).

5. Eugenia, patricienne d'Alexandrie, a reçu une haute instruction. Ses parents veulent la marier, à quoi elle se dérobe en quittant la maison sous des habits d'homme avec ses deux eunuques, Protus et Hyacinthus, qui sont chrétiens. 
Ils arrivent au monastère de l'abbé Hélénus qui leur donne à tous trois la vêture, quoiqu'il sache à quoi s'en tenir sur le prétendu frère Eugène, lequel devient prévôt de l'église et fait des miracles. Une femme guérie par Eugenia s'éprend d'elle et, devant sa résistance, l'accuse d'avoir voulu lui faire violence. Eugenia est traînée en justice devant son propre père qui commence par ne pas la reconnaître. Une reconnaissance émouvante est suivie d'une conversion générale. Eugénie est revêtue de vêtements dorés et tenue en grand honneur avant de finir martyrisée (Delehaye, Etude sur le légendier romain, saints de nov. et déc., 1936, p. 171 ; Légende dorée, I, p. 380).

6. Apollinaria, que ses parents veulent marier à l'empereur, aspire à la vie religieuse et s'enfuit avec un eunuque et un vieillard. Elle achète à une vieille des vêtements monastiques qu'elle prend, laissant les siens à ses deux compagnons qui les renvoient aux parents, lesquels la pleurent comme morte. Elle vit longtemps en anachorète, dans un marais dont les moustiques la dévorent. Une voix l'avertit, si on lui demande qui elle est, de répondre : Dorotheus. Elle obtient de saint Macaire, qui voit en elle un eunuque, l'autorisation d'habiter au monastère. Elle y fait des miracles et, notamment, guérit sa propre sœur possédée du démon qui l'a toujours détestée. Mais le démon donne à cette sœur l'apparence de la grossesse et fait accuser le moine. Dorotheus est jugée par son propre père. Après une reconnaissance générale elle rentre au couvent où son sexe véritable n'est révélé qu'après sa mort (fête le 5 janvier, Acta, janvier I, p. 258).

7. Euphrosyne d'Alexandrie est la fille tard-venue de Paphnuce qui, resté veuf, la fait instruire soigneusement et souhaite la marier. Elle aspire à la vie monastique, mais elle sait que si elle entre dans un couvent de femmes son père, qui l'aime, viendra l'y rechercher. Sur le conseil d'un moine, elle se déguise en homme et dit à l'abbé qu'elle est Smaragdos, eunuque du palais. Comme elle est fort belle et que le diable inspire aux autres frères des désirs coupables, l'abbé la fait 
mettre dans une cellule anachorétique où elle vit paisiblement. Cependant son père désolé la fait rechercher dans tous les couvents de femmes et demande des jeûnes et des prières aux frères parmi lesquels elle vit. Un jour ils se trouvent même face à face, mais il ne reconnait pas son visage altéré par les austérités, qu'elle cache sous son manteau. Elle vit de la sorte pendant trente-huit ans et ne se fait reconnaitre qu'en mourant. Une châsse contenant ses reliques était conservée à Saint-Jean-aux-Bois près de Compiègne (fête le 11 février, Acta, février II, p. 535 ; Analecta Bollandiana, t. II, 1883, pp. 196-205).

8. Matrona de Pergé, quoique mariée et mère d'une fille, aspire à la vie monastique. Elle se voit en songe poursuivie par un homme et se réfugiant parmi des moines. Elle se fait alors couper les cheveux, prend l'habit d'eunuque et entre au monastère de saint Bassian sous le nom de l'eunuque Babylas. Bassian, averti par un songe, interroge Matrona qui se disculpe : son mari l'empêchait par coups et reproches de suivre sa vocation; elle a pris soin de confier sa fille à une femme de bien; enfin, un rêve l'a conduite au monastère. L'évêque éprouve pour elle une vive admiration, mais l'avertit de se couvrir dorénavant la tête, " conformément à la loi naturelle ". Après de nombreuses péripéties, elle revient au monastère pour y vivre en ascète et faire des miracles (Patr. grecque, t. CXVI, p. 920).

9. Théodora d'Alexandrie, ayant cédé au démon qui l'incitait à tromper son mari, va expier sa faute dans un monastère d'hommes où elle fait les plus durs travaux. Accusée par une fille enceinte, elle est chassée du cloître puis réadmise, tant sa pénitence est édifiante, avec l'enfant dont on lui attribue la paternité. Son sexe n'est révélé qu'à sa mort; son mari et l'enfant se font moines à leur tour (fête 11 sept., Acta, sept. III, p. 788 ; Légende dorée, I, p. 150).

10. Anastasia, patricienne de Constantinople, est aimée de l'empereur, auquel elle n'accorde rien. Fuyant la jalousie de l'impératrice, elle se réfugie dans un couvent de femmes. 
L'empereur devenu veuf la fait rechercher. Elle part de nuit et va exposer son cas à l'abbé Daniel qui lui donne des vêtements masculins et l'enferme dans une grotte où, sous le nom d'Anastasius eunuchus, elle vivra sans que personne puisse l'approcher, excepté le frère qui lui apporte de l'eau. Après vingt-huit ans d'austérités, se sentant mourir, elle fait appeler l'abbé Daniel et le prie de l'enterrer sans la dénuder. Mais le disciple qui la met au tombeau voit sa poitrine nue et s'étonne. Le travestissement, lui dit l'abbé, était le seul moyen d'échapper à l'empereur (honorée le 10 mars, Acta, mars II, p. 40).

11. Marie l'Égyptienne expie au désert une vie de péché. L'abbé Zosime la découvre nue, son vêtement étant tombé en lambeaux depuis quarante-sept ans qu'elle vit là. A sa prière, il la revêt de son propre manteau. L'année suivante, il revient lui apporter la communion pascale et la trouve morte (honorée le $1^{\mathrm{er}}$ avril, Acta, avril I, p. 68 ; Légende dorée, II, p. 63). Même épisode dans la vie de sainte Théoctiste, qui n'est qu'un doublet de Marie l'Égyptienne (H. Delahaye, Sanctus, 1927, p. 221 sqq).

12. Papula, Gauloise, voudrait être religieuse, mais ses parents l'aiment trop pour y consentir. Elle entre à Tours, à leur insu, dans un couvent d'hommes, et y brille par de si grandes vertus qu'à la mort de l'abbé on l'élit à sa place ; elle ne révèle son sexe que trois jours avant de mourir (Grégoire de Tours, Gloria confessorum, 16).

13. Hildegonde, de Nuitz près de Cologne, est vêtue en garçon et nommée Joseph par son père, en vue d'un voyage à Jérusalem. Le père meurt en mer. Après de nombreuses aventures, un ange la sauve du gibet et l'envoie, toujours travestie, à l'abbaye cistercienne de Schönau. L'abbé et les moines sont miraculeusement aveuglés sur son sexe véritable, révélé seulement lorsqu'elle meurt en 1188 , en odeur de sainteté1.

1) 20 avril, Acta, avril II, p. 782 ; sa vie, censément racontée par un de ses frères en religion, s'écarte fortement du schéma courant; on ne peut étudier ici les problèmes qu'elle implique, ni celui de la pénétration en Occident d'un thème essentiellement grec. 


\section{II. - Travestissement temporaire}

14. Thekla, ayant quitté sa mère et son fiancé pour suivre saint Paul, se fait couper les cheveux, s'habille en homme et, dans cet appareil, se rend chez Tryphaena à Mérou. Cet épisode, qui ne joue aucun rôle dans la suite du récit, manque dans la plupart des recueils édifiants; la Légende dorée, II, 334, dit que Thècle voulut se couper les cheveux mais que "l'apôtre ne le permit pas car elle était d'une grande beauté "(fête 23 sept., Acta, sept. IV, p. 546).

15. Glaphyra, vierge, veut éviter le mariage et la poursuite de Licinius, gouverneur du Pont qui, contrairement aux ordres de Constantin, persécute les chrétiens. La reine Constance, sœur de Constantin et femme de Licinius la fait évader en vêtements masculins. Elle meurt avant d'être reprise par Licinius (honorée le 13 janvier, Acta, janvier II, p. 53).

16. Une femme qui voudrait consulter saint Siméon Stylite se déguise en soldat et se joint à d'autres pèlerins, mais n'ose entrer dans sa maison. Dès que les autres abordent le saint, celui-ci les charge de dire " au soldat qui est resté dehors que sa prière est exaucée ". La femme alors confesse qu'elle est une pécheresse (Acta, janvier I, p. 267).

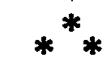

A Pélagie, à Marina, à Marguerite, Usener joignait une Anthusa, une Porphyria, dans les noms desquelles il reconnaissait des épithètes, grecques ou latines, d'Aphrodite, la Marine, la Porteuse de Perles, la Fleurie, la Pourprée. En elles toutes, il voyait " les rejets d'un arbre coupé », de l'Aphroditos bisexué de Chypre, auquel les fidèles sacrifiaient, les femmes en vêtements d'hommes, les hommes en vêtements de femmes. Mais ces noms ne sont que des épithètes rares et littéraires de la déesse, et les correspondances entre les dates des fêtes sont plus que douteuses. Usener retrouvait aussi un souvenir d'Aphroditos dans les saintes barbues, lesquelles ne 
doivent rien à l'antiquité, comme le montrait fort bien, dès 1867, Ch. C. Cahier dans ses Caractéristiques des Saints (I, p. 121). Usener aimait creuser profond pour trouver ses explications; que ceux qui croient ne rien lui devoir lui jettent la première pierre ${ }^{1}$.

Partant de la légende de sainte Thècle, L. Radermacher voit dans le roman grec l'origine du thème. Margarita en effet se comporte exactement comme, dans les Ephesiaca de Xénophon, Thelxinoé qui aime Aigialeus alors qu'on veut la forcer à en épouser un autre. Aigialeus l'enlève de la chambre nuptiale en habits d'homme, après lui avoir coupé les cheveux. Erwin Rohde (Der griechische Roman, p. 385) avait rappelé que, dans le rite lacédémonien du mariage (Plut., Lyc., 15) la nympheutria rase la tête de la mariée et étend celle-ci en vêtements masculins sur le lit nuptial, où l'époux vient la rejoindre dans l'obscurité. Aigialeus après s'être déguisé en nympheutria aurait profité du travestissement momentané de l'épouse pour la faire évader. Comme Thelxinoé fuit un fiancé qu'elle déteste, ainsi Margarita fuit le mariage qu'elle a en horreur. La ressemblance s'arrête là, puisque le vêtement masculin, temporaire pour la première, est définitif pour la seconde.

Mais comment ramener aux dimensions d'un simple procédé littéraire un thème d'une valeur psychologique si riche et si constante ? On s'étonne qu'Hippolyte Delehaye, indépendamment de Radermacher, se soit satisfait de voir dans la sainte en habits d'hommes un thème du folklore oriental, une variation romanesque dans l'histoire de la courtisane convertie ${ }^{2}$. Si cela était, le thème apparaitrait-il

1) H. Usener, Legenden der Pelagia, Bonn, 1879, où l'on trouvera le classement des traditions et l'indication des sources pour les légendes 1 à 14 ; L. RaderMACher, Hippolytos u. Thekla, Akad. Wiss. Wien, 1916, t. CLXXXII, fasc. 3.

2) Les légendes hagiographiques ${ }^{3}, 1927$, pp. 177 et 192 sqq. Pour le P. Delehaye, l'histoire a été inventée pour donner du piquant à l'histoire d'une courtisane d'Antioche, mentionnée dans l'homélie LXVII de Jean Chrysostome. Après son baptême, cette femme vécut de longues années sous le cilice, sans se laisser voir de personne. Cette emprisonnée volontaire serait devenue Pélagie dont toutes les autres ne sont que des répliques littéraires. 
dans l'histoire avec une signification identique à celle qu'il a dans la légende ?

Jeanne d'Arc prend l'habit d'homme, et c'est trop peu de dire qu'elle le garde jusqu'à la mort. Elle en meurt. Lorsqu'on voit l'importance du travestissement dans l'acte d'accusation, on se dit que ses juges auraient été cruellement embarrassés si, moins obstinée, elle leur avait enlevé ce grief.

La mystique Antoinette Bourignon, née en 1616, eut très jeune des extases au cours desquelles elle s'évanouissait. Le Seigneur lui dit : "Soyez plus virile; je suis pur esprit, insensible à la chair. "Ses parents voulant la forcer à se marier, le jour de Pâques 1636 elle part de Lille à 4 heures du matin sous un habit d'ermite qu'elle s'est fait elle-même. Un peu après Mons, comme elle avance difficilement, les pieds meurtris par ses gros souliers de paysan, des soldats la reconnaissent pour une femme. On la loge à Blaton chez le maire où un officier tente de la violer. Le curé de Blaton la cache chez lui et fait venir l'archevêque qui lui représente les dangers de la vie d'ermite et l'erreur qu'elle a faite en prenant ce costume.

Jeanne d'Arc, brûlée puis canonisée, Antoinette Bourignon, pauvre sainte manquée, eurent certainement des sœurs qui, elles aussi, se comportèrent comme Thècle ou Pélagie. Ces deux exemples peuvent suffire; rapprochés des précédents et des contes de la sainte barbue, ils permettent une analyse et des conclusions.

\section{$*^{*} *$}

Les légendes grecques ont-elles un fond de réalité ? Leur parfaite invraisemblance pourrait être imputable aux narrateurs qui les ont excessivement embellies. A une époque où il $\mathrm{y}$ avait des eunuques, où certains chrétiens se mutilaient dans l'excès de leur ferveur, une femme pouvait, à la rigueur, être prise pour l'un d'eux et comme tel vivre en anachorète, ce que du reste elle pouvait tout aussi bien faire en se donnant pour ce qu'elle était. Dans ce cas, le travestissement est gratuit. Il l'est du reste tout autant lorsqu'il s'agit de vie 
monastique et, en plus, concrètement inimaginable. La connivence du supérieur ne joue aucun rôle dans nos récits ; elle est acquise à Pelagia, à Eugenia, à Anastasia, tandis que Marina, Apollinaria, Euphrosyné, Theodora, Hildegonde, cachent leur sexe véritable à l'abbé qui les accueille. Bassian, d'abord induit en erreur par Matrona, se rend ensuite à ses raisons et entre dans le jeu. Dans la mesure où s'accuse le caractère romanesque du sujet, les auteurs cherchent à donner au travestissement une justification raisonnable : Euphrosyné et Papula se réfugient parmi les moines parce que leurs parents, partout ailleurs, les feraient rechercher. Anastasia fuit la poursuite de l'empereur, Matrona se dérobe à son mari. Cette dernière légende est la seule où l'abbé fasse un reproche à la pénitente et se souvienne des interdictions de saint Paul : "Toute femme qui prie ou qui prophétise la tête non voilée déshonore sa tête, comme si elle était rasée... A cause des anges, la femme doit avoir sur la tête un signe de sujétion... La nature elle-même nous l'enseigne : la chevelure lui a été donnée en guise de voile "(Cor., I, II, 4-11). Matrona se disculpe en disant qu'elle empêchait son capuchon de retomber quand elle allait à la communion.

Ni Bassian, ni aucun autre abbé ne parait se souvenir de l'interdiction du Deutéronome : "Une femme ne portera pas un habit d'homme et un homme ne mettra point un vêtement de femme, car celui qui fait ces choses est en abomination à Jéhovah son Dieu "(22,5). Malheureusement pour Jeanne d'Arc, les docteurs de Rouen eurent meilleure mémoire.

Aucun des récits ne comporte le moindre blâme à l'égard de femmes qui se comportent avec un parfait mépris pour la discipline. En dépit de leur apparente humilité, toutes se sentent supérieures aux lois écrites. Plusieurs d'entre elles paient cher le rejet de la condition féminine ; aucune cependant ne regrette son choix au point de revenir en arrière. Les récits les plus anciens concernaient certainement non des moines, mais des ermites. Et il est vrai que, comme l'a dit Louis Duchesne (Histoire ancienne de l'Église, II ${ }^{4}$, p. 491), 
l'anachorète est en soi la critique vivante de la société ecclésiastique. Cela, les rédacteurs des Vies, qui manquaient de recul, ne l'ont pas senti. En revanche, ils auraient pu et logiquement ils auraient dû censurer l'imprudence de ces abbés qui accueillent des femmes déguisées et juger plus sévèrement encore celles qui induisent les supérieurs en erreur et les frères en tentation. Non seulement ils ne l'ont jamais fait, mais dans trois légendes le faux eunuque arrive dans le couvent à une haute situation : Margarita se voit confier un monastère de religieuses. Eugenia devient prévôt, Papula est élue abbé. Confrontée à ces histoires, la fable de la papesse Jeanne semble être à la fois un passage à la limite, et une satire du genre tout entier ${ }^{1}$. Mais, avec cette fable, on quitte le monde monacal pour entrer dans la hiérarchie ecclésiastique et c'est un tout autre univers.

Les vies des saintes ermites et moines se sont influencées l'une l'autre, et l'imagination des conteurs les a enrichies de

1) Les premières versions écrites connues sont du milieu du xirre siècle. Au $x^{\circ}{ }^{\bullet}$, la légende est universellement admise, au point qu'au début du xve le buste de la papesse figure dans la cathédrale de Sienne parmi ceux des pontifes. Pie II, Pie III et Marcel II, papes siennois, ne s'en offusquèrent point. C'est Clément VII I (1592-1605), qui le fit retailler pour qu'elle représentât le pape Zacharie. La légende dérive probablement de plusieurs usages et monuments mal interprétés, dont le principal est la porphyretica, fauteuil archaïque au siège échancré qui servait aux intronisations, et auquel on attribua un usage imaginaire : ubi dicitur probari papa an sit vir. Cette première invention en fit naître une autre, à savoir que l'imposture d'une femme aurait rendu la probation nécessaire. Le raisonnement de Döllinger sur ce point et sur plusieurs autres est convaincant (Die Papstfabeln des Mittelalters, 1863, p. 27). Mais l'élaboration de l'histoire ne s'explique pleinement que parallèlement à celle des saintes travesties. Celles-ci entrent dans des couvents, d'où les ennuques n'ont jamais été exclus. En revanche, ils le sont et l'ont toujours été du clergé séculier où le moindre défaut physique est une clause d'exclusion. Une femme-abbé est donc, à la rigueur, imaginable ; une femmepape ne l'est pas. Son aventure doit donc se terminer par un échec. Mais afin que l'échec soit satisfaisant pour l'esprit il faut que, de plus, la femme soit répréhensible. On l'imaginera donc quittant son couvent pour suivre un moine et, pour finir, accouchant dans la rue et succombant à la honte. On retrouve donc ici, retournés, les thèmes des Vies : la passion coupable remplace la chasteté et, bien loin d'échapper pour toujours à la condition féminine, la papesse est vaincuê par la nature et de la façon la plus humiliante. Et cependant, malgré ses fautes et la sanction finale, Jeanne, dans la plupart des récits, est représentée comme pieuse et savante, allant s'instruire en Grèce, fondant des écoles, formant des disciples. Elle reste parente d'Eugénie et de Catherine, leur sour présomptueuse et condamnée. Je ne pense pas qu'on ait jamais mis sa légende en rapport avec celles des saintes travesties, bien faites cependant pour l'éclairer, à la fois par des similitudes et par des contrastes. 
motifs adventices. Deux fois, le travestissement permet à une femme de rejoindre dans la vie religieuse un homme qui lui est cher. Marina se fait moine pour ne pas quitter son père, Athanasia, pour vivre sous le même toit que son mari, lequel du reste ne la reconnaît pas. Les deux récits sont d'une égale absurdité. Eugenios une fois mort, sa fille n'a plus aucune raison d'éterniser un stratagème qui ne peut que la mettre en difficulté. Mais, précisément, le narrateur voulait introduire la péripétie de l'innocence calomniée (qui figure également dans les vies de Margarita, Eugenia, Theodora, et, relevée d'ingénieuses subtilités, dans celle d'Apollinaria). Tant que vit Eugenios, Marina pouvait être immédiatement disculpée ; pour qu'elle fût obligée de souffrir en silence, il fallait que le père fût mort. Quant à Athanasia, elle se déguise avant de partir en pèlerinage parce qu'elle doit être méconnaissable au moment où elle rencontre son mari. Dans ces deux romans, une composition à la fois travaillée et maladroite concilie vaille que vaille la primauté de l'ascèse (et tout ce que celle-ci comporte ipso facto d'hostilité à l'égard de la famille) avec le respect des sentiments familiaux représentés par un père et sa fille, par des époux chrétiens tendrement attachés l'un à l'autre. Pour Hildegonde, une fois le pèlerinage terminé, le travestissement est d'une gratuité absolue.

Dans toutes les histoires, le travestissement signifie rupture avec l'existence antérieure. Celle-ci peut être une vie coupable (Pelagia, Theodora, Marie l'Égyptienne, la visiteuse anonyme de saint Siméon); ou l'insistance d'un homme impudent (Anastasia, Glaphyra); ou un mariage déjà consommé (Matrona); ou des fiançailles près d'être consacrées (Margarita, Apollinaria, Thékla); ou enfin, le vœu pressant de parents désireux de marier leur fille contre sa volonté (Eugenia, Euphrosyné, Papula, Jeanne d'Arc, Antoinette Bourignon).

La rupture avec la vie antérieure est soulignée par une hostilité plus ou moins grande à l'égard de la famille. Margarita, Apollinaria fuient simplement la leur. Thékla n'a pas de pire 
ennemie que sa mère. Les juges de 1431 ont reproché à Jeanne son impietas erga parentes : elle n'avait trouvé en eux qu'incompréhension et mauvais traitements. Antoinette Bourignon avait un bec de lièvre et sa mère ne put jamais surmonter l'aversion que lui inspirait sa laideur. Dans les légendes. d'Eugenia, Euphrosyné, Papula, le narrateur a essayé de concilier la rupture avec les bons sentiments ; on voit comment il y a réussi.

Dans un grand nombre de vies de saintes, un changement de vêtements apparaît chargé d'une valeur symbolique. Une Pelagia qui n'est pas la nôtre, jeune vierge de quinze ans, sur le point d'être prise et violentée par des soldats, revêt pour se tuer sa robe de mariée : fertur nuptialem induisse vestem, ut non ad mortem ire diceres sed ad sponsum, dit d'elle saint Ambroise (De Virginibus, III, 7, 34). Une troisième Pelagia enlève au moment du baptême ses vêtements précieux et en fait présent à l'évêque qui lui donne une robe monastique ${ }^{1}$ à la vue de laquelle sa mère la dénonce aux persécuteurs. Même épisode dans la vie de sainte Anthusa (22 août, Acta, août IV, p. 499). Thaïs brûle au moment de sa conversion les parures qu'elle a reçues pour prix de son corps ( 8 oct., Acta, oct. IV, p. 225). Sainte Catherine de Bologne porte toujours les habits d'une pauvresse, mais elle les soigne pour recevoir l'Eucharistie. Elle apparait un jour miraculeusement entourée d'une troupe de beaux jeunes gens qui la parent comme une reine d'une robe blanche toute semblable au pallium que les prêtres revêtent pour les cérémonies solennelles. Ce symbole du sacerdoce est le salaire de son obéissance, que ses supérieurs ont éprouvée en lui ordonnant de se jeter dans le feu (elle le fait, mais on la retient), puis de se mettre nue pour aller chez sa mère, et on l'arrête comme elle se dévêtait (9 mars, Acta, mars II, pp. 73, 43-4, 37). Il est difficile de croire à la réalité de ces deux expériences. Le baptême du feu et le dénudement

1) La Vie grecque (chap. IX) présente ici une lacune; H. UsEner, Legenden der Pelagia, p. 22 ; H. Delehaye, Légendes hagiographiques, p. 187. 
sont des rites d'initiation. Sainte Perpétue se voit en rêve transportée dans l'amphithéâtre, dépouillée de ses vêtements et transformée en homme : le symbole est ici particulièrement net, mais, comme nous le verrons, il s'y mêle des thèmes accessoires.

De tous les changements d'habits, le plus frappant est celui qui suggère un changement de sexe, L'hagiographie a retrouvé là un rite archaïque dont elle a curieusement gauchi le sens. Dans les initiations de la protohistoire hellénique, les jeunes garçons admis dans la classe des adultes mettent puis enlèvent solennellement des robes de filles, symbole de l'essence féminine qui a baigné leur enfance et avec laquelle ils rompent définitivement en devenant des hommes faits'. Un rite nuptial comme celui de Sparte, où la fiancée est habillée en garçon, donne à penser qu'un travestissement existait aussi dans les initiations féminines, mais celles-ci ont tôt perdu toute importance et n'ont laissé que des vestiges incertains. Tout ce qu'on peut dire, c'est que, dans le milieu hellénique et romain où s'est répandu le christianisme primitif, il existait :

a) Plusieurs légendes de garçons habillés en filles (Achille, Leucippos, Hyménée, Dionysos) ;

b) Quelques légendes de filles habillées en garçons (Leucippé, Procris) ;

c) Des rites assez nombreux où le prêtre et les fidèles hommes s'habillent en femmes, censément à l'imitation de leur dieu (Héraclès de Cos, Hercule Victor romain, cortège dionysiaque) ;

d) Quelques traces peu sûres de rites où des officiantes revêtaient des attributs masculins (fêtes lacédémoniennes d'Artémis).

En somme, à la réserve des "carnavals " qui comportaient un échange réciproque (Hybristika d'Argos, culte chypriote

1) H. Jeanmaire, Couroi et Couretes, pp. 153 et 321 . Le travestissement avait, je pense, un sens plus riche et devait conférer à chacun des deux sexes quelque chose des puissances de l'autre. Sur ce point et sur les travestissements antiques, voir mon livre Hermaphrodite, Presses Universitaires de France, chap. Ier. 
d'Aphroditos), le cas le plus fréquemment attesté est celui des hommes en vêtements de femmes.

L'hagiographie donne un tableau tout opposé. On n'y trouverait pas aisément un parallèle masculin aux saintes travesties; ou, plus exactement, celui-ci correspond à ce que serait, chez les hommes, l'éviration. Dans les initiations antiques, on enlève le vêtement féminin pour renoncer à la féminité, symbole encore parfaitement vivant dans l'hagiographie. Mais il ne s'agit plus ici de garçons qui se détachent du monde féminin où ils ont vécu. Ce sont des jeunes femmes qui renoncent à leur propre sexe pour devenir, comme le disent leurs biographes avec complaisance, des eunuques. Passent pour tels Pelagia, Marina, Apollinaria, Matrona, Euphrosyné, Anastasia. Eugenia s'enfuit avec deux eunuques qui s'associent à son triomphe. Si, comme je le pense, l'échange des vêtements a signifié pour les jeunes Grecs une sorte d'acquisition symbolique de la puissance humaine sous ses deux aspects, pour les jeunes chrétiennes il signifie renoncement au sexe. Cela n'a rien qui doive beaucoup nous étonner, puisque déjà chez Ovide androgynie est synonyme d'asexualité.

Un renoncement identique est explicitement formulé dans les histoires de la sainte barbue. Plusieurs études ont élucidé l'origine de ce conte, inspiré par la vue de crucifiés en tuniques analogues au Volto Santo de Lucques, honoré dès le $x_{I I}{ }^{e}$ siècle ${ }^{1}$. Pour qu'on y ait vu une femme, il a fallu que cette représentation archaïque d'un Jésus au visage immobile, à la robe longue, aux yeux ouverts, couronné d'un diadème, contrastât fortement avec les représentations de plus en plus réalistes et pathétiques $d u$ Christ nu aux reins ceints $d u$ perizonium, au visage tourmenté, aux yeux clos, au front déchiré par les épines. L'interprétation fémininine du Volto Santo a donné lieu à plusieurs légendes que voici, dans l'ordre croissant de leur élaboration romanesque.

1) Biblioggraphie chez. H. Del.enaYF, Légendes hagiographiques, p. 103, n. 4 ; J. Gessler, La Vierge barbue, sainte Wilgeforde, Bruxelles, 1938, étudie l'aire de lispersion et l'iconographie. 
1. Galla, fille de Symmaque, veuve romaine, souhaite ne pas se remarier. Les médecins lui font craindre que l'excessive chaleur de son corps, privée de tout exutoire, ne fasse pousser une barbe, ce qui arrive en effet. Sed sancta mulier nihil exterioris deformitatis timuit quae interioris sponsi speciem amavit. Elle meurt d'un cancer au sein (5 octobre, Acta, oct. III, p. 162).

2. Paula, vierge d'Avila, poursuivie par un prétendant, se jette au pied d'un crucifix et supplie le Christ de la défigurer. Elle est aussitôt exaucée : barba agrestis enata, frons distorta, genae foedatae. Son poursuivant passe à côté d'elle sans la reconnaître (Acta, févr. III, p. 174).

3. Wilgeforte (virgo fortis) est la fille d'un roi païen de Lusitanie qui menace de la marier au roi de Sicile alors qu'elle veut garder sa virginité. A sa prière, le Christ lui fait pousser une barbe. Furieux de se résistance, son père la fait crucifier. On l'appellera dorénavant Liberata (20 juillet, Acta, juill. IV, p. 50$)$.

Gessler considère la légende comme originaire des PaysBas et datant $\mathrm{du} \mathrm{xv}^{\mathrm{e}}$ siècle. Ce qui nous intéresse ici, c'est l'extraordinaire pullulation d'un culte qui garde partout une valeur unique. Comme le vêtement masculin, la barbe signifie renoncement au sexe. Dans l'histoire de Galla et de Paula, où ne figure pas encore le thème étiologique de la crucifixion, le renoncement est symbolisé par le cancer au sein, par le visage méconnaissable aux yeux du prétendant. La barbue crucifiée est la Libérée, sainte Livrade en France, Librada en Espagne (retable du Xvi ${ }^{\text {e }}$ siècle et prétendu tombeau dans la cathédrale de Siguënza). sainte Débarras à Beauvais, Ohnliummer en Allemagne, Ontcommere en Flandre, Uncumber en Angleterre. La dénomination elle-même fit inventer des prolongements légendaires : on disait en Angleterre que la sainte était secourable aux femmes qui voulaient se débarrasser de leurs maris ${ }^{1}$. La défiguration par la barbe a la même

1) Ch. (. Autwn, Garacterisliques des saints, 1867. I, p. 121 . 
valeur que la lèpre dont sainte Enimia obtint d'être frappée ob virginitatis custodiam (6 oct., Acta, oct. III, p. 406), que les mutilations que s'infligent sainte Lucie, qui s'arrache les yeux, sainte Eusébie, qui se coupe le nez. De plus, la transformation s'accompagne d'une vive hostilité à l'égard de la famille, hostilité qui se marque encore dans les superstitions de l'hagiographie populaire.

\section{$*^{*} *$}

Antoinette Bourignon n'avait aucune culture, mais elle avait pu lire, ou entendre raconter d'après la Légende dorée les histoires de Marguerite, de Thècle, d'Eugénie qui revêtent des habits d'ermite pour fuir leur famille. Elle a dû s'y complaire. Son père était despotique et brutal. Sa mère, qui ne l'aimait pas, la maltraitait. Les psychanalystes connaissent bien les névroses qui résultent chez les filles du " complexe anti-maternel ". Elles se manifestent souvent par un refus de la vie sexuelle. Alles, nur nicht wie die Mutter ${ }^{1}$. Le déguisement en ermite, considéré comme une ruse pour passer inaperçue, était une absurdité. Mais c'était pour elle infiniment plus : un symbole dont la suite de sa vie atteste l'importance. Elle enseigna que Dieu avait créé l'homme à la fois mâle et femelle, capable de se reproduire lui-même, comme les plantes ; c'est le péché qui nous a fait déchoir de cet état idéal, nous imposant la conjonction et la douleur. On dit qu'elle vit en extase la beauté du monde et d'Adam tels qu'il étaient avant la chute. Ces rêveries permettent de dépasser la signification impliquée dans nos légendes, car le refus de la vie sexuelle y apparaît comme une aspiration à une androgynie transcendante au péché. Une image semblable existe dans la gnose et dans le mysticisme juif, avec une valeur identique.

1) C. G. Jung, Von den Wurzeln des Bewusstseins, Zürich, 1954, p. 109. — Les tragiques grecs ont ratifié la formule en refusant toute vie sexuelle à Électre ennemie de sa mère. Sur ce point comme sur d'autres, Euripide a cherché à innover, mais sans arriver à persuader. - Sur. Antoinette, voir S. Reinach, Cultes, Mythes religions, I, p. 421, et la thèse, en suédois, de John Björkhem, Upsal, 1940, pp. 3847 et 390. 
Est-il permis de croire que sur Jeanne d'Arc tout n'a pas été dit ? que Thècle et Pélagie peuvent jeter sur elle quelque lumière ?

Au procès de réhabilitation, Jean de Metz dit que c'est lui qui, à Vaucouleurs (janvier 1429), lui proposa de prendre habit d'homme, ce qu'elle accepta aussitôt, sur quoi il lui donna les vêtements et les chaussures d'un.de ses famuli ${ }^{1}$. A son retour de Nancy, quelques habitants de Vaucouleurs lui firent faire un accoutrement masculin; elle se coupa les cheveux en rond et Baudricourt lui donna une épée qu'elle remplaça plus tard par celle de l'église Sainte-Catherine à Fierbois, à l'entrée de la Touraine.

Dix fois, à Rouen, on l'interrogea sur ce point. Jamais elle ne répondit par l'argument du bon sens, mais s'enveloppa de mystère.

Interrogatoire du 22 février : "Requise de dire par quel conseil elle avait pris habit d'homme, elle refusa plusieurs fois de répondre. Finalement dit que de cela elle ne chargeait personne et plusieurs fois varia... Dit encore qu'il lui avait fallu (oportuerat; Champion : " Qu'il lui fallait néces" sairement ") changer son habit en habit d'homme. Elle sait bien que son conseil le lui a dit "(Champion, I, p. 40; II, p. 35).

27 février : "Interrogée si Dieu lui a prescrit de prendre habit d'homme, répondit que l'habit c'est peu de chose, la moindre... Elle ne. l'a pris par conseil de qui que ce soit, mais par commandement de Dieu et de ses anges... Interrogée si elle l'a fait par ordre de Robert de Baudricourt, répondit que non "(Champion I, p. 55 ; II, p. 49).

1) Témoignage concordant de Poulengy. J. Quicherat, Procès... de Jeanne $d^{\prime} A r c, 1844$, II, p. 436 ; le procès de réhabilitation occupe les t. II et III ; pour celui de Rouen, v. P. Champion, Procès de condamnation de Jeanne d'Arc, 1920-21 ; t. I, texte. t. II, traduction (souvent assez libre). 
Même question, même réponse le 12 mars, puis ceci : "Interrogée si la voix lui commanda qu'elle prit habit d'homme, répondit : Tout ce que j'ai fait de bien, je l'ai fait par le commandement de mes voix ; et quant à l'habit, j'en répondrai une autre fois ; de présent n'en suis point avisée " (I, p. 104 ; II, p. 81).

14 mars. "Sur ce qu'elle porte habit d'homme, répondit : Puisque je le fais par le commandement de notre Sire et en son service, je ne crois point mal faire, et, quand il Lui plaira de le commander, il sera aussitôt mis bas "(I, 129; II, 95).

Ce que le réquisitoire traduit : "Les dites Catherine et Marguerite commandèrent à cette femme, de par Dieu, qu'elle prit et portât l'habit d'homme ; et elle l'a porté et le porte encore, obéissant audit commandement avec obstination, au point de déclarer qu'elle aimerait mieux mourir que de délaisser ledit habit ; disant parfois cela sans plus et ajoutant parfois : à moins que Dieu ne le commande "(I, 271-274; II, 207-209).

Les réponses de Jeanne concernant son retour, en prison, au costume féminin, sont d'une grande imprécision. Au début du procès, elle avait demandé à entendre la messe, ce qui lui avait été refusé, vu les crimes dont elle était accusée et l'inconvenance de son habillement. Le 15 mars on lui met le marché à la main : prendre habit de femme et ouïr la messe ou demeurer en habit d'homme et ne pas ouïr la messe. Elle se débat : "Certifiez-moi que j'ouïrai messe si je suis en habit de femme et sur ce je vous répondrai. - Je vous le certifie. Et que dites-vous si j'ai juré et promis à notre roi de ne pas abandonner cet habit? Toutefois, faites-moi faire une robe longue pour aller à la messe et puis, au retour, je reprendrai l'habit que j'ai. " "Interrogée si elle prendrait cet habit une fois pour toutes, répondit : je me conseillerai sur ce et puis vous répondrai. En outre dit le plus instamment qu'elle put qu'elle requérait qu'on lui permît d'ouïr la messe dans l'habit qu'elle portait, sans en changer" (I, p. 132; II, p. 97).

Le refus plus net du 27 mars (" si on lui donnait congé de 
s'en aller en habit de femme, elle se mettrait aussitôt en habit d'homme et ferait ce qui est commandé par NotreSeigneur "I, p. 143 ; II, p. 102) a peut-être été obtenu par les juges qui tenaient à ce grief majeur. Toutefois, l'acte d'accusation du 5 avril (I, p. 271 ; II, p. 207) et l'admonition du 2 mai (I, pp. 317-321 ; II, pp. 246-250) consignent un fait exact en disant que Jeanne a renoncé à la messe et à la communion pascale plutôt que de changer d'habit. De quelques marchandages que résulte cette décision, une chose paraît certaine : pas une seule fois Jeanne n'allègue l'argument utilitaire, à savoir que le vêtement masculin, plus commode pour une femme-soldat, était mieux fait également pour protéger une prisonnière quoique, à vrai dire, la complicité des gardiens eût permis à n'importe qui de lui faire violence, quel que fût son vêtement. Tout se passe comme si l'habit était pour elle un pur symbole, mais de la plus haute importance. Elle reprend à l'abjuration une robe de femme apportée par son confesseur; le $24 \mathrm{mai}$, à la révocation, elle remet ses anciens habits qu'on lui avait laissés dans un sac, probablement pour la tenter, et alors seulement donne comme raison sibi magis conveniens habere habitum virilem dum erat inter viros (I, 374; II, 289).

Trois des membres du tribunal, le notaire Guillaume Manchon et les dominicains Isambart de La Pierre et Martin Ladvenu témoignèrent en 1450 l'avoir entendue se plaindre à ce moment d'outrages subis en prison (Quicherat, II, $5,8,365$; III, 148-9). La chose est trop vraisemblable. Mais si l'argument a joué un rôle dans la détermination de Jeanne, ce ne put être que dans les derniers jours de sa vie.

Au début du procès, elle ne pouvait mesurer la gravité de l'accusation. Des prêtres l'avaient entourée en Lorraine, son parent Henri de Vouthon, curé de Sermaize, le curé Jean Fournier qui s'assura par la formule de l'exorcisme qu'elle n'était pas mue par le démon, son confesseur Jean Colin qui, fin février 1429, assista à son départ en compagnie de Bertrand 
de Poulengy et de Jean de Metz. Aucun ne lui reprocha son accoutrement, et pas davantage les clercs qui l'interrogèrent à son arrivée à Chinon. Le réquisitoire dit (Champion, I, p. 181 ; II, p. 131) que Baudricourt désapprouva le travestissement cum magna abominatione, ce qui ne résulte d'aucun interrogatoire; mais si Jeanne mit tant de soin à mettre Baudricourt hors cause, c'est peut-être en effet qu'elle avait rencontré chez lui une certaine résistance. A Poitiers, des théologiens l'examinèrent pendant six semaines et, entre autres choses, voulurent savoir pourquoi elle avait changé d'habits. C'étaient des docteurs armagnacs, ils ne durent pas insister beaucoup.

Deux ans plus tard, le 3 mars, les juges de Rouen voulurent savoir ce qu'avaient dit leurs collègues, mais Jeanne ne s'en souvenait plus (Champion, I, p. 71 ; II, p. 63). Or, elle semble avoir répondu à Poitiers tout autrement qu'à Rouen. Les docteurs en effet décidèrent qu'il fallait l'autoriser à garder l'habit masculin puisqu'elle l'avait pris, non pour offenser la pudeur d'autrui, mais pour garder la sienne. Voilà de nouveau l'argument raisonnable, celui que Jean de Metz avait mis en avant deux mois auparavant, que les trois clercs repentants alléguèrent en 1449 , avec dix-huit ans de retard, mais que Jeanne, à Rouen, à notre connaissance, ne mentionna jamais.

Sut-elle même que les théologiens de Poitiers, soutenus par Jean Gerson dont ce fut là le dernier écrit, avaient décidé à son bénéfice que l'interdiction du Deutéronome n'avait pas une valeur absolue ? Elle ne pouvait pas deviner l'importance que le problème prendrait dans sa propre vie, et ces discussions, à supposer qu'on lui en ait dit quelque chose, durent lui paraitre oiseuses. Les docteurs rappelèrent le synode de Gangres en Paphlagonie où, vers 340, Basile de Césarée attaqua les excès ascétiques d'Eustathe de Sébaste, lequel imposait des accoutrements bizarres à ses fidèles, hommes et femmes. Le synode condamna Eustathe et rappela que les femmes ne doivent porter vêtements d'homme ni se 
couper les cheveux ${ }^{1}$. Contre cette décision, les docteurs rappelèrent les saintes qui, inspirées par un mouvement extraordinaire de l'Esprit-Saint, avaient caché leur sexe sous des vêtements virils, telle sainte Euphrosyné d'Alexandrie, révérée à Saint-Jean près de Compiègne. Gerson, qui allègue la Camille de Virgile et les Amazones - précédents fort intéressants pour nous, mais peu probants pour des théologiens - n'a pas pensé à sainte Euphrosyné. Il voyait en Jeanne une guerrière et non une sainte et l'idée ne lui vint pas qu'elle pût avoir été influencée par la légende des pénitentes travesties. Comme les docteurs de Poitiers, il limitait l'interdiction du Deutéronome à l'habit indécent. Aucun d'eux n'utilisa à fond l'argument décisif apporté à leur thèse par ces vies que la tradition unanime donnait comme exemplaires et auxquelles les siècles suivants continuèrent de donner une approbation totale. Dieu ne montre jamais mieux sa puissance, dit le Commentarius praevius à la vie de Marina (Acta, juill. IV, p. 278), que par le courage qu'il donne à de tendres vierges, lesquelles deviennent ainsi capables de presque dépasser les saints par leurs austérités et leur patience. "Je veux dire ces vierges ou ces femmes qui, se donnant pour des moines, nous ont laissé des exemples d'actes héroïques capables de nous remplir tous d'admiration ; si périlleux toutefois qu'on ne saurait conseiller de les imiter. Démentant leur sexe, si l'on peut dire, elles ont continuellement vécu parmi des moines, endurant jusqu'à leur mort des injures et des reproches dont elles auraient pu se disculper si la nature n'avait été vaincue en elles par cette grâce dont une ineffable Providence avait comblé les âmes qu'elle s'était choisies. "Des considérations analogues introduisent la vie d'Hildegonde. On ne saurait homologuer avec plus de décision un acte qui, en toute rigueur de termes, relève de l'indiscipline et de la dissimulation. La Légende dorée accorde aux saintes traves-

1) La consultation de Gerson figure dans Quicherat, III, pp. 298-306 ; le texte du Synode et les interprétations de plus en plus catégoriques du canon 12 sont dans Mansi, Sacrorum Conciliorum Collectio, II, 1095 et suiv. 
ties la même louange sans nuance. Bien plus, si Paul, dit-elle, empêcha Thècle de se couper les cheveux, ce n'est pas à cause des authentiques interdictions qu'il formula en ce sens, c'est parce qu'elle était d'une grande beauté, argument de romancier, qui aurait inspiré à saint Paul un grand étonnement et une violente colère. Une jeune fille nourrie de ces histoires, pouvaitelle s'attendre à l'intransigeance des juges de Rouen?

Comme Antoinette Bourignon, Jeanne est psychologiquement de la famille des pénitentes travesties. Pour elle seule en face de toutes les autres, le travestissement eut une valeur pratique. Mais il signifiait beaucoup plus encore, à savoir exactement ce qu'il avait signifié pour elles, et même des choses qui, pour Jeanne elle-même, restaient plus qu'à demi inconscientes.

La confrontation des légendes nous révèle le sens symbolique du travestissement : rupture avec le passé féminin, hostilité à l'égard de la famille et de l'autorité, renoncement à la vie sexuelle. Jeanne a quitté Domrémy sans esprit de retour. Elle était bien décidée à ne jamais se marier. Sur ce point, elle n'a jamais douté qu'elle n'eût raison d'agir comme elle faisait. Mais son expérience comportait un point douloureux : ses rapports avec sa famille. Cette enfant à qui l'on avait répété : tes père et mère honoreras, pouvait se sentir à la fois lésée et coupable, car aucune affection semble n'avoir existé entre elle et ses parents.

Lorsque son oncle Durand Laxart la mena une première fois à Vaucouleurs, Baudricourt lui dit : "Reconduis-la à son père avec de bons soufflets. "Jacques d'Arc déclara de plus que si Jeanne recommençait il la ferait noyer par ses frères, réponse typique de cruel lâche (Champion, I, p. 103; II, p. 81). Elle passa alors deux semaines à Neufchâteau où un garçon de Domrémy l'assigna devant l'official pour rupture de promesse de mariage. Bien entendu, les parents d'Arc tinrent avec le garçon, celui-ci représentant la vie normale à quoi ils souhaitaient ranger leur fille. C'est malgré leur défense qu'elle soutint son procès et comparut devant l'official. 
A partir du second départ pour Vaucouleurs, Jacques d'Arc semble ne plus s'être occupé de sa fille. Aucun de ses parents ne demanda à être entendu comme témoin au procès. L'imposture de la dame des Armoises, cinq ans après la mort de Jeanne, aurait été impossible sans la complicité des frères d'Arc et peut-être même de la mère, laquelle vivait encore ainsi que Jean, prévôt de Vaucouleurs, et Pierre, pris à Compiègne avec sa sœur et libéré après une longue captivité. Tous avaient été anoblis le 29 décembre 1429.

Comme dans les légendes grecques de la femme ermite, l'horreur du mariage et l'hostilité à l'égard de la famille sont liées l'une à l'autre. Rien d'étonnant si, dès le premier interrogatoire (22 février, Champion, I, p. 39 ; II, p. 34), Jeanne déclare que la voix lui ordonnait de partir sans que son père sût rien de son départ. Elle agit comme Euphrosyné et Papula qui, elles, aimaient leur père et néanmoins le firent souffrir pour suivre leur vocation. Jeanne put entendre parler d'Euphrosyné à Compiègne où elle séjourna après le sacre en août 1429, de Papula à Tours. La légende synonyme d'Eugénie est dans la Légende dorée ainsi que celles de Marina, Théodora, Pélagie et des deux Marguerite.

Les historiens de Jeanne, parlant des Voix qui la guidèrent, citent saint Michel, les saintes Catherine et Marguerite comme si tous trois avaient été constamment à ses côtés depuis Domrémy jusqu'au bûcher. Une lecture attentive des deux procès impose ici des distinctions. Jeanne parla d'abord d'une Voix qui lui apparut et lui ordonna de partir (Champion, I, p. 105 ; Quicherat, III, p. 204). Dunois et le duc Jean d'Alençon s'exprimèrent de même en 1455. Elle dit ensuite que ce fut saint Michel qui l'envoya à Baudricourt et lui ordonna de faire sacrer le dauphin. Saint Michel, patron du duché de Bar, était en passe de devenir celui du royaume tout entier, d'autant plus que le Mont-Saint-Michel, courageusement défendu, était avec Tournai la seule ville du nordouest qui fût restée fidèle au roi. Dunois parla en 1455 d'une vision qu'elle aurait eue de saint Denis et de Charlemagne : 
témoignage isolé d'un homme qui remplace probablement de mémoire un nom par deux autres dont la signification psychologique lui paraît équivalente. A l'interrogatoire du 1 er mars, Jeanne déclara n'avoir plus vu saint Michel depuis le Crotoy (fin novembre 1430). Ge victorieux, ce rayonnant, n'avait plus rien à dire à une prisonnière vaincue.

A Rouen, où saint Michel ne joue plus aucun rôle (sauf à la minute suprême), Jeanne est constamment assistée par ses saintes. Or, au procès de 1455, aucun des compagnons de la Pucelle ne mentionne même leur nom, sauf une fois, pour dire qu'elles la réconfortèrent lorsqu'elle fut blessée à Orléans (Quicherat, III, p. 110). Tout se passe comme si elles entraient en scène au moment de l'épreuve et pour aider leur fille spirituelle à la surmonter. Voyons ce qu'elles représentaient pour la bergère devenue chef de guerre.

La Légende dorée loue deux Marguerite. La première (I, p. 153) vit loin de son père, prêtre païen, garde les troupeaux, est poursuivie par Olybrius, combat le diable et lui dit : "Tremble, ennemi superbe, tu gis sous les pieds d'une femme "; elle échappe à tous les supplices avant de mourir décapitée. La seconde (II, p. 178) est le no 2 de notre propre inventaire ; elle fuit à la fois ses parents et un mariage dont elle ne veut pas. Déguisée en homme elle se réfugie dans un monastère. C'est la première de ces deux Marguerite, invoquée par les femmes en couches, dont le culte fut populaire en France depuis le moyen âge. Il n'est pas impossible que Jeanne ait aussi connu la seconde et que, peut-être à son insu, le thème du travestissement ait coloré la première.

Quant à Catherine (Légende dorée, II, p. 207), elle dispute victorieusement avec l'empereur qui fait venir cinquante orateurs pour défendre contre elle la cause des faux dieux. Ils se convertissent à sa parole et reçoivent le baptême du sang. Le tyran ébloui offre à Catherine la première place après l'impératrice. Elle refuse, sort intacte et victorieuse de tortures incroyables, après quoi elle finit par être décapitée. Conclusion habituelle de ces légendes, ce supplice en somme 
humain et rapide parait presque dénué de réalité après les atrocités auxquelles il succède. Catherine était honorée à Maxey tout près de Domrémy, où Jeanne, dont la sœur s'appelait Catherine, a dû entendre raconter son histoire.

Jeanne trouvait en saint Michel la transfiguration même de son rêve; Marguerite et Catherine la représentaient elle-même, telle qu'elle était, telle qu'elle aurait voulu être. Catherine possède les pouvoirs qui, à Rouen, lui auraient été les plus précieux : le don de persuader qu'elle-même avait exercé au temps de ses victoires mais qui restait sans effet sur des juges bourguignons; et, plus encore, la science qui lui aurait permis de discuter avec des théologiens, espèce qu'elle avait toujours détestée précisément parce qu'en leur présence elle sentait cruellement son infériorité. Marguerite et Catherine, faibles femmes, remportent un triomphe total sur des hommes qui commencent par les mépriser. Jeanne souffrait du dédain avec lequel les docteurs abordaient les mulierculae. D'Estivet, le promoteur de 1431, l'appelait paillarde et ordure et c'était pour la bonne règle, car il savait qu'elle était vierge. Dans l'agressivité de Jeanne, il y a de l'orgueil blessé. La blessure, soyons-en certains, date des mauvais traitements de Jacques d'Arc. Les juges de Poitiers, qui ne furent pas bien durs avec elle, s'entendirent répondre : "Oui, je crois en Dieu, et mieux que vous... Il y a ès livres de Notre-Seigneur plus qu'ès vôtres "(Quicherat, III, p. 86), ce qui implique peut-être moins de mépris pour les livres des clercs que de regret de ne pouvoir les ouvrir. Saint Michel a pu être pour elle un modèle pendant l'année glorieuse de sa carrière, et d'autant plus que dans la Légende dorée il apparaît à sainte Catherine pour la réconforter avant son combat avec les docteurs. Mais, pendant son enfance méprisée, à Poitiers où sainte Catherine vint la rassurer (Quicherat, III, p. 82) et surtout pendant les mois sombres du procès de Rouen, Marguerite et Catherine furent pour elle un Wunschbild. G'étaient des femmes délivrées de la condition féminine qu'elle haïssait, ainsi qu'en témoigne son mépris pour Cathe- 
rine de La Rochelle : "Qu'elle retourne vers son mari faire son ménage et nourrir ses enfants" ( 3 mars, Champion I, p. 80 ; II, p. 68) ; et, cette condition, elle l'aurait probablement mieux acceptée si ses rapports avec ses parents avaient été meilleurs. Les deux saintes, c'étaient des femmes débarrassées de tous liens familiaux, éloquentes et sûres d'elles, assez fortes pour se faire respecter des hommes avec l'aide de Dieu et pour sa cause. "Si elle refuse l'habit de femme c'est parce qu'elle veut rester chef de guerre et qu'elle compte sur la promesse des voix " dit Anatole France (Jeanne d'Arc, II, p. 305). Ou, plutôt, c'est parce qu'elle ne peut renoncer à être chef de guerre qu'elle ne cesse d'invoquer ses saintes. Penser à elles nourrissait à la fois son courage et ses illusions. C'est pourquoi l'on comprend qu'après l'abjuration, puis en recevant la communion avant de mourir, elle ait dit que ses voix l'avaient déçue. Le Wunschbild lui avait montré un triomphe suivi d'une mort sans réalité, une fin presque abstraite. D'après le dominicain Jean Toutmouillé (1455), elle dit qu'elle aimerait mieux être décapitée (comme Catherine et comme Marguerite) sept fois que d'être brûlée. Et alors, ne sentant plus entre elle et ses saintes la parenté qui l'avait soutenue, elle revint à' saint Michel et l'invoqua au moment où on la liait ${ }^{1}$.

Comment Jeanne n'a-t-elle jamais allégué pour sa défense l'exemple des saintes qui prirent le vêtement masculin? C'est peut-être qu'elle ne sentait pas une ressemblance suffisante entre elle, guerrière, et ces ascètes. C'est peut-être aussi par une sincère humilité, ne voulant pas se comparer aux élus de Dieu. Cependant, son attitude pendant le procès était d'une extraordinaire audace. Jeanne répond un peu comme les martyres qui traitent magistrats et empereurs avec une égale arrogance. On pourrait s'attendre à trouver parfois, dans la littérature des Actes, quelque jeune fille

1) Témoignages de Pierre Boucher et de Guillaume de La Chambre en 1455. PÉguY dans sa première Jeanne d'Arc (1897, CEuvres complètes, t. XV, p. 699) a très bien senti la valeur de ce moment : Mes sœurs du Paradis qui m'avez renoncée, et qui me laissez seule... J'étais la sœur humaine et vous les sours célestes; j'étais la scur plus jeune el vous les deux aînées. 
timide qui prouve sa foi en allant silencieusement à la mort. Des "dernières à l'échafaud " il dut y en avoir sous Dioclétien comme pendant la Terreur. Aucun narrateur ne parait avoir senti ni l'intérêt psychologique ni l'intérêt dramatique du contraste. Les chrétiennes persécutées sont d'une éloquence terrifiante et toujours victorieuse. Là encore, une pauvre enfant chaque jour plus menacée trouvait une image idéale sur laquelle elle essaya de se modeler jusqu'au jour où la réalité contredit douloureusement sa chimère.

\section{$*^{*}{ }^{*}$}

Les aventures des vierges qui, pour échapper à la persécution, revêtent l'habit masculin peuvent avoir subi l'influence du roman grec. La figure des saintes en froc monacal laisse peut-être deviner, mais c'est bien douteux, le vague souvenir d'une divinité bisexuée. La dévotion aux saintes barbues trouve son explication immédiate dans un crucifix mal interprété. Mais, par delà les sentiments chrétiens, de possibles rémanences païennes et des images qui remontent aux initiations archaïques, c'est dans la psyché qu'il faut chercher les racines d'une association entre travestissement masculin d'une part et d'autre part hostilité envers la famille, rupture avec la vie antérieure et avec l'ordre établi, renoncement à la vie sexuelle, refus des disciplines établies. Ces légendes rapprochées de quelques épisodes historiques d'une curieuse synonymie prouvent la valeur autonome de symboles assez puissants pour s'introduire presque sans transposition dans des récits dont aussitôt ils dirigent l'économie. Bien plus : pour s'imposer à la femme de chair qui vit l'aventure au point de faire corps avec elle.$$
*^{*} *
$$

Les deux cas historiques que nous venons de considérer sont assez clairs. Au moment où Antoinette Bourignon choisit de rompre avec sa famille, le vêtement masculin fut pour elle le symbole même de la rupture. Lorsque Jeanne à Vaucouleurs 
s'accoutra en homme d'armes, c'était pour une raison de commodité qui, en prison, n'existait plus. Si elle a si dangereusement tenu à cet habit dont on lui faisait grief, c'est que, pour elle aussi, il avait une valeur symbolique, transcendante à tout usage pratique. On voit mal comment il aurait pu protéger concrètement une fille enchaînée contre les brutalités de ses gardiens. Mais pour elle et peut-être même pour eux il était synonyme de ce nom de Pucelle qu'on lui donna dès son entrée en action et qu'elle laissa aussitôt substituer à celui de son père. Ses compagnons d'armes et de voyages déclarent unanimement qu'elle ne leur inspira jamais aucun désir. Il est permis de croire que ce qui inhibait en eux la voix de la nature, c'est le renoncement impliqué par son habit, joint à la conviction qu'elle était chargée de pouvoirs mystérieux. Ses geôliers de Rouen en furent peut-être également intimidés. L'habit était comme le signe visible de la rançon dont elle avait payé ces pouvoirs.

Dans les débuts de la vie anachorétique, quand, en Palestine et en Syrie, les solitaires vivent à distance les uns des autres, sans supérieur qui les dirige, on peut admettre à la rigueur qu'une pécheresse repentante, décidée à mener cette vie, ait marqué par l'habit masculin qu'elle répudiait la cause même de ses péchés. Son cas serait psychologiquement analogue à celui d'Antoinette Bourignon. Or, le directeur de Pélagie, Nonnos, est de la règle de saint Pacôme. Celui-ci, au début $\mathrm{du} \mathrm{Iv}^{\mathrm{e}}$ siècle, donna en Égypte la première règle pour la vie en commun des hommes qui renonçaient au monde, tandis que sa sœur fondait des monastères féminins. Un disciple de ce législateur aurait dû s'opposer au projet de Pélagie. Le récit grec de Jacques, publié par Usener qui le date du second quart du ve siècle, escamote avec une étrange habileté tout ce qui touche à la responsabilité de Nonnos. Celui-ci préside à la conversion et au baptême. Puis, après un intervalle de trois ans, le narrateur retrouve la pénitente sans la reconnaitre sous l'aspect de cet " eunuque solitaire " muré dans sa cellule. 
Mais, dès qu'il s'agit de vie monastique, le " changement de sexe "n'est plus possible sans la connivence positive de l'entourage. Et comment imaginer un abbé autorisant une femme à vivre sous le froc parmi des moines, ou, davantage, la supercherie une fois découverte, lui permettant de continuer une existence aussi anormale ? L'histoire de Pélagie peut être considérée comme un passage à la limite à partir d'un fait authentique. Les autres sont des légendes plus ou moins romancées. Où trouver l'origine du thème qui leur sert de centre?

C'est, je pense, dans les doctrines et les pratiques rigoristes du christianisme naissant. On prêcha alors le renoncement total aux biens matériels et à toute vie sexuelle, même dans le mariage. Saint Paul s'est élevé contre cet ascétisme qu'il jugeait excessif (première épître à Timothée). Mais la gnose, païenne, juive ou chrétienne, devait reprendre la tendance et l'accentuer, en enseignant que la matière, essentiellement mauvaise, a été créée par un démiurge destiné à être vaincu par un dieu bon. Sans être gnostiques, les Actes apocryphes de Paul (dont l'histoire de Thècle n'est qu'un épisode), ceux de Jean, de Pierre, d'André, de Thomas, exigent du chrétien un ascétisme continuel et détournent violemment du mariage. Le rigorisme atteint son apogée avec les Encratites, les continents par excellence, qui refusent toute participation à l'œuvre du démiurge. Pendant le second tiers du ive siècle, Eustathe de Sébaste reprend leurs doctrines. Il refuse aux riches tout espoir de salut s'ils ne se débarrassent de la totalité de leurs biens; il conseille à ses adeptes de quitter leur famille et de fuir les villes; il leur impose un accoutrement bizarre, le même pour les hommes et pour les femmes, lesquelles se coupent les cheveux; surtout, il interdit le mariage. Le concile de Gangres blâma ces pratiques où il voyait une critique de la vie religieuse telle que l'Église la menait. En effet, bien des prescriptions d'Eustathe paraissent dictées par l'intention délibérée de rompre avec les usages courants : il détournait de faire des offrandes à l'Église, recomman- 
dait de jeûner le dimanche et de manger aux jours de jeûne.

Il fut condamné peu après par le concile de Nicée (325) lequel, à bien des égards, représente le triomphe de la pondération occidentale sur l'Orient hellénisé. C'est à Nicée qu'il fut interdit de conférer les ordres aux eunuques volontaires, alors que, vers 200 , Origène qui venait de se mutiler avait été ordonné prêtre par deux évêques palestiniens.

La tendance ascétique, qui survécut aux blâmes des conciles, inspire toutes nos légendes. Telles que nous les lisons, elles ne formulent que des sentiments orthodoxes. Interrogées avec quelque patience, elles laissent transparaitre l'idéologie condamnée à Gangres. Comme la pousse de la barbe dans la légende de sainte Liberata, la prise de l'habit masculin est, pour une femme, l'équivalent de la castration. Pour comprendre le prestige religieux de celle-ci, il faut se souvenir du rôle que tient le concept de l'androgyne dans toutes les formes, grecques ou hébraïques, de la gnose. Androgynie à la fois initiale et finale. Dans sa perfection édénique, l'homme avait les deux puissances; il les recouvrera dans son ascension suprême. C'est par cette conception que s'expliquent probablement les surnoms mystiques au masculin qui figurent parfois sur des sépultures de femmes : la morte aspirerait à avoir, comme son dieu, les deux natures. Encore faut-il objecter qu'on n'a trouvé nulle part de surnoms mystiques féminins sur des sépultures d'hommes ${ }^{1}$. La bisexualité des philosophes équivaut à une asexualité : l'homme spirituel est totalement libéré des liens de la chair. S'il est impossible de ratifier l'hypothèse d'Usener voyant dans la sainte travestie une résurgence de l'Aphroditos chypriote, il reste que la clef de la légende est l'image gnostique d'une androgynie idéale.

L'image avait une telle autorité qu'on la trouve même dans des textes orthodoxes. Sainte Perpétue a une vision au

1) J. Carcopino, Le mystère d'un symbole chrétien, 1955, p. 45, et Aspects mystiques de la Rome païenne, $2^{\circ}$ éd., pp. 275 et 284. 
cours de laquelle elle se voit dans l'amphithéâtre : et expoliata sum vestibus et facta sum masculus (Acta, mars I, pp. 633-8). Ce texte a peut-être une portée moins générale qu'il ne paraît d'abord. Le rêve concerne les préliminaires d'un combat contre les démons; Perpétue s'y voit sous l'apparence d'un gladiateur. Encore la victoire finale y est-elle liée à l'enlèvement des vêtements féminins et au changement de sexe.

Pélagie est anachorète. L'anachorète, qui se passe des sacrements et qui échappe à toute discipline, représente dans la société ecclésiastique le triomphe de l'individu sur la règle. Dépouillée de la littérature pieuse qui les enveloppe, nos légendes donnent une leçon d'anarchie. Elles prônent très exactement ce qui fut condamné à Gangres, c'est-à-dire des tendances que l'Église a refrénées sans jamais pouvoir les supprimer totalement. Leur hétérodoxie apparaît dans la mesure même où leurs auteurs prétendent enseigner la docilité la plus stricte. La sainte désexuée devient moine : concession à la régularité. Mais on ne pouvait se la représenter dans une communauté masculine sans envisager du même coup les complications qui résulteront du stratagème. D'où, avec passage à la limite, l'épisode de l'innocente accusée d'une paternité coupable. Si la vraisemblance réglait le récit, l'inculpée n'aurait qu'un mot à dire pour établir la vérité. Ce mot, elle ne le prononce jamais; et aucun narrateur ne lui reproche d'avoir causé du scandale et déshonoré le couvent par son silence. La logique est dans un autre plan, qui n'est pas celui du réel. La femme qui a renoncé au sexe est accusée d'avoir péché par le sexe. Aucune accusation ne pouvant être plus imméritée, c'est celle qu'il est le plus méritoire de supporter en silence.

Un certain sadisme affleure dans la complaisance des narrateurs à détailler les humiliations qui frappent la calomniée. Elle a prétendu abdiquer un sexe méprisé et appartenir au sexe supérieur : qu'elle accepte les conséquences de son audace. Jamais, à coup sûr, le travestissement n'est explicitement blâmé. A mesure qu'ils cessaient d'en comprendre la 
valeur symbolique, les remanieurs ont déployé une ingéniosité croissante pour l'étayer de motifs raisonnables : la sainte agit louablement pour dérouter des poursuites coupables. Des histoires si bien agencées mettent leur héroïne au-dessus de tout reproche. Mais l'inconscient des conteurs, plus souvent qu'ils ne le pensaient, allait dans le même sens que le conscient des juges de Rouen. C'est pourquoi ils décrivent sans déplaisir les épreuves par lesquelles la femme-moine achète son triomphe final et les tortures qui précèdent la libération de la barbue crucifiée. Et une étrange cruauté affleure dans le passage où Aruns, sterneret ut subita turbatam morte Camillam, perce la guerrière d'un trait qui s'enfonce profondément dans le sein nu et fait couler le sang virginal. Le double sens de la blessure $\mathrm{y}$ est évident. Image analogue à la victoire d'Achille sur l'Amazone : une femme domptée git à terre, à la merci du vainqueur.

Marie Delcourt. 